\title{
VIABILIDAD DE LA INTERMODALIDAD EN EL TRANSPORTE DE PERECEDEROS
}

\author{
JUAN CARLOS PÉREZ MESA \\ Departamento de Economía y Empresa UNIVERSIDAD DE ALMERÍA, ESPAÑA \\ e-mail: juancarl@ual.es \\ LUCIA ABALLAY \\ Departamento de Economía y Empresa UNIVERSIDAD DE ALMERÍA, ESPAÑA \\ e-mail: lucia@inlumine.ual.es
}

\section{RESUMEN}

Este trabajo trata de estudiar la viabilidad de la intermodalidad en el transporte de productos hortícolas desde el sureste de España. Un sector que genera, sólo en exportación, casi 3.000 millones de $€$. En Almería, referente del sector hortícola de España en producción y exportación hacia la Unión Europea, prácticamente la totalidad del tráfico se realiza por camión frigorífico. Ante el aumento tendencial de su coste, las trabas futuras para su utilización (tasas ambientes o limitación de tránsitos) y la dependencia estratégica del sector productor-exportador de ese sistema, se hace necesaria la búsqueda de fórmulas logísticas alternativas. En este sentido, la intermodalidad se presenta como una opción, entendida como el movimiento de mercancías, en una misma unidad de carga, utilizando dos o más medios de transporte. En concreto, en este trabajo se comprueba el potencial de la logística terrestre-marítima combinando camión refrigerado y buques Ro-Ro, con el objetivo de aumentar la competitividad de las empresas de comercialización hortofrutícola mediante la definición de alternativas logísticas que, de forma paralela, permitan reducir el impacto ambiental del transporte. Los resultados muestran que las rutas hacia Reino Unido son las mejor posicionadas para el cambio modal.

Palabras clave: hortalizas, logística, camión, transporte marítimo.

\section{Viability of intermodal transport for perishables}

\begin{abstract}
This work studies the viability of intermodal transport applied to horticultural products from the south-east of Spain. This sector has an exportation turnover of 3,000 million $€$. All Almeria's transportation, which is the most important horticultural province in Spain, is carried by refrigerated truck. Given the trend increase of cost, the future obstacles to its use (environmental rates or transit limitations) and the strategic dependence of the exporter sector, it is necessary to search for alternative logistic formulas. In this sense, intermodal transport is a good option, understood as the movement of goods, in the same cargo unit, using two or more means of transport. This work analyzes the current routes in operation using refrigerated truck and Ro-Ro vessels. The results show that the routes from southeast of Spain to United Kingdom are the most viable.
\end{abstract}

Keywords: vegetables, logistics, truck, maritime transport

Clasificación JEL: F18, Q17 


\section{INTRODUCCIÓN}

El transporte representa una parte importante de la economía de la Unión Europea y es un sector estratégico: emplea a casi 10 millones de personas y representa cerca de un 5\% del PIB (Eurostat, 2017). A pesar de su relevancia, la estructura del sector está en cuestión, especialmente el tránsito terrestre. Las políticas de la UE durante los últimos años reflejan un contexto de cambio estructural y retos a los que el sector del transporte por carretera se enfrenta, ya que la Comisión Europea tiene un compromiso claro con la implementación de la "movilidad limpia". Recientemente, la Comisión ha tomado varias iniciativas para fomentar el desarrollo del Espacio europeo del transporte, una política que ha ido persiguiendo durante décadas. De forma concreta, se han introducido las primeras normas de la historia sobre emisiones de $\mathrm{CO} 2$ aplicables a los vehículos pesados, promoviendo un sistema de movilidad con bajas emisiones (Comisión Europea, 2018). En 2025, las emisiones medias de CO2 de los camiones nuevos tendrán que ser un $15 \%$ más bajas que en 2019. Para 2030, se ha propuesto un objetivo de reducción de, como mínimo, un $30 \%$ con respecto a 2019. También se ha propuesto lograr una transferencia modal del $50 \%$ del transporte por carretera al ferroviario y marítimo en el caso del transporte de mercancías.

El sector de análisis de este trabajo es la horticultura española, con especial mención a Almería como principal provincia productora y exportadora. Concretamente, se analiza el transporte de hortalizas (perecederos) desde Almería hacia la Unión Europea y la viabilidad de un cambio modal con empleo del transporte marítimo de corta distancia (TMCD). La Unión Europea es el mercado fundamental de Almería representando el 98,7\% del total exportado (Figura 1): Alemania es el principal destino con el $32,5 \%$ del total, seguido de Francia (15\%) y Reino Unido (12\%). En la actualidad, la exportación supone el destino del $76 \%$ de la producción (Cajamar, 2017). Respecto al canal de venta, destaca que la gran distribución europea (Aldi, Edeka, Tesco, Carrefour, Lidl, etc.) absorbe el 70\% de las compras a los productores españoles (Pérez-Mesa \& Galdeano, 2015), gestionando alrededor del 80\% de los tránsitos. En conjunto, el 30\% de todas las hortalizas compradas por el consumidor europeo provienen de Almería (Serrano-Arcos, 2018).

\section{Figura 1}

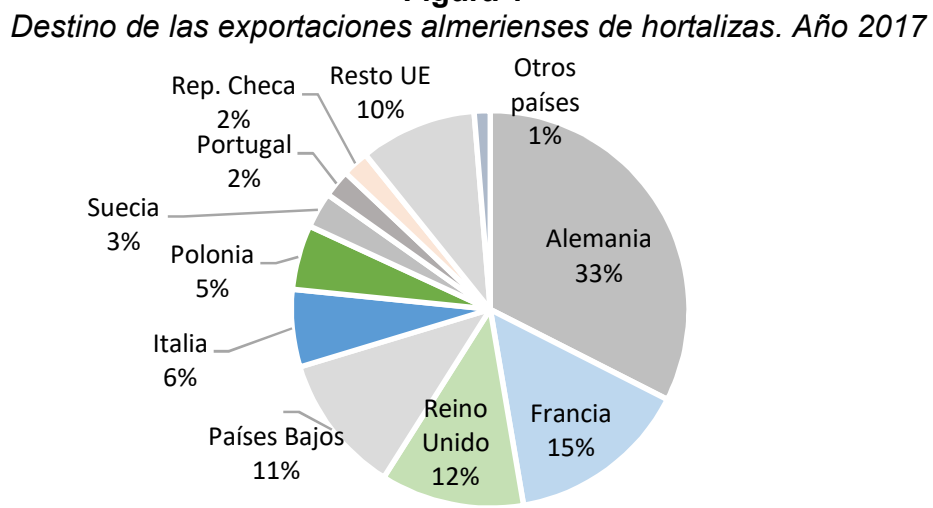

Fuente: ICEX (2017)

Actualmente, el transporte por carretera es la solución más adoptada para cumplir con las expectativas de conservación de los perecederos en transporte internacional (hasta $98 \%$ en el caso de Almería). La combinación de la flexibilidad, la velocidad, la transparencia, la sencillez y la inexistencia de problemas de cruce de fronteras hace que el transporte por carretera, especialmente en las distancias cortas, sea una modalidad con la que es difícil competir (den Boer et al., 2011). Como punto negativo, existe una tendencia creciente de su coste, al margen de las bajadas coyunturales de los últimos años (Figura 2). Hay que tener en cuenta que en una transacción internacional el transporte puede suponer del $20 \%$ al $30 \%$ del coste total, siendo el concepto de mayor relevancia después de descontar la mano de obra incluida en las diferentes fases del proceso (García-Barranco y Pérez-Mesa, 2015), lo que demuestra la necesidad de buscar alternativas y/o complementos a este modo.

La opción intermodal desde el sureste de España para productos hortofrutícolas ha sido estudiada en diversos trabajos (Pérez-Mesa et al., 2010; Pérez-Mesa y Galdeano-Gómez, 2012), todos ellos partían 
de la premisa de crear líneas ad hoc que pudieran hacer más factible el cambio modal. Como novedad este trabajo se centra en desarrollar rutas que ya funcionan en la actualidad por lo que la rapidez de implantación, una vez tomada la decisión, sería más rápida.

Figura 2

Evolución del coste $(€ / \mathrm{km})$ de un vehículo frigorífico articulado de 2 ejes cargado

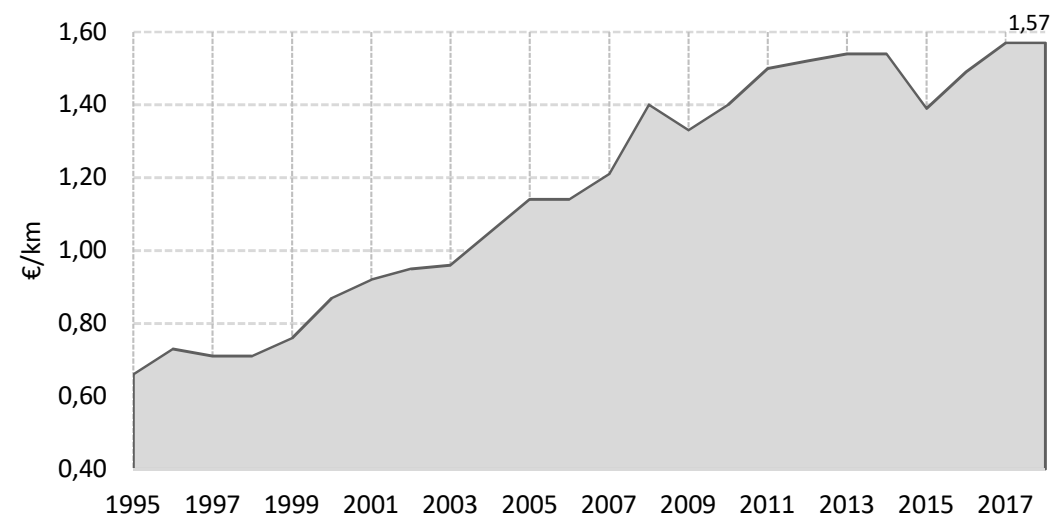

Fuente: Elaboración propia a partir de datos del Ministerio de Fomento (2017).

\section{INTERMODALIDAD Y TMCD}

La intermodalidad es entendida como el movimiento de mercancías en una misma unidad de carga, utilizando dos o más modos de transporte sin manipulación de la mercancía al cambiar de medio, y donde la mayor parte de la ruta se realiza por ferrocarril o vía navegable (López et al., 2015). Se presenta como el sistema de organización logística con mayor eficiencia y respeto al medio ambiente y se desarrolla en una serie de etapas (Paixao y Marlow, 2009): i) recogida en la zona de origen y transporte por carretera a la terminal intermodal, ii) transbordo de la mercancía al siguiente medio de transporte empleado (ferroviario, marítimo, aéreo), iii) transporte de la carga entre las terminales intermodales de origen y destino; iv) transferencia de los productos a un camión; v) y distribución por carretera desde la terminal al lugar final de destino de la mercancía.

El principal objetivo de este sistema es aprovechar las fortalezas de distintos medios de transporte en una cadena integrada, optimizando el rendimiento económico. Sin embargo, para que la intermodalidad sea considerada una alternativa al transporte por carretera de larga distancia, los costes generales tienen que ser iguales o inferiores, y los costes adicionales, causados por el pre y posttransporte, así como los de trasbordos en las terminales intermodales, deben ser compensados (Hanssen et al., 2012).

Además, no solo existen beneficios económicos sino también ambientales, puesto que al combinar distintos medios y utilizar el más adecuado en cada parte del viaje, el impacto ambiental se reduce (Paixao y Marlow, 2009). Por ello, desde la Unión Europea la intermodalidad se ha incluido en los diferentes Libros Blancos del Transporte como una estrategia prioritaria, con la que hacer frente a la congestión de las principales rutas de transporte, disminuir la emisión de $\mathrm{CO} 2$, y reducir la siniestralidad (Comisión Europea, 2001 y 2011).

En España, siguiendo la línea marcada por la UE, la intermodalidad forma parte de las directrices de actuación del Plan Estratégico de Infraestructuras y Transporte 2020, tanto para el transporte de pasajeros como de mercancías (Ministerio de Fomento, 2005). En relación con este último, algunos objetivos son: i) la mejora de la capacidad intermodal de los puertos y de sus accesos ferroviarios; ii) la consolidación de la red intermodal de plataformas logísticas y centros de mercancías en cooperación con el resto de las administraciones públicas, operadores y del sector privado; iii) la creación de corredores multimodales de transporte de mercancías que complementen a los corredores tradicionales; iv) el desarrollo de las autopistas del mar.

Hoy en día, existen una multitud de líneas marítimas regulares que conectan los Estados miembros de la UE entre sí y con otros países ribereños, en un tipo de navegación conocido como Transporte 
Marítimo a Corta Distancia (TMCD) o Short Sea Shipping (SSS). Este, se entiende como el movimiento de mercancías y pasajeros por mar entre puertos situados en territorio de la Unión Europea o entre esos puertos y puertos situados en países no europeos con una línea de costa en los mares que rodean Europa, según la normativa comunitaria y la legislación española. Durante las últimas dos décadas, la Unión Europea ha liderado la promoción de corredores marítimos como alternativa al transporte por carretera. Se ha señalado como la motivación principal la necesidad de establecer un campo de juego equitativo entre los modos de transporte, así como de reducir la congestión y otros daños ambientales causados por el transporte por carretera (Suarez-Alemán, 2016).

Este sistema de transporte forma parte de las cadenas marítimo-terrestres y su competitividad depende de que se cubran todos los niveles de calidad y precio que impone la demanda de transporte (Medda y Trujillo, 2010). Además, para que sea eficaz y eficiente deben existir cadenas alternativas razonables de transporte ferroviario y/o por carretera. En consecuencia, el concepto de TMCD en Europa se basa también en la competencia entre las cadenas marítimo-terrestres y las exclusivamente terrestres (García Barranco et al., 2016).

El concepto de TMCD está vinculado con las denominadas Autopistas del Mar (AdM), rutas entre determinadas fachadas marítimas en las que es posible establecer cadenas logísticas, con procedimientos administrativos y de aduanas simplificados, e introducir sistemas comunes de gestión del tráfico. Es decir, las AdM parten de la idea de crear una "vía" permanente en el mar, estableciendo rutas con un horario y puertos de origen y de destino definidos, que no serán cambiados a corto plazo. Surgen con una opción de transporte "puerta a puerta", suponiendo la combinación del TMCD con el transporte por carretera y el uso del camión para los eslabones de recogida y entrega (Suárez-Alemán et al., 2014).

El potencial del transporte por ferrocarril y marítimo apenas ha sido explotado en el caso de las frutas y hortalizas debido a la reticencia de los exportadores a la utilización de otros medios distintos al camión, sobre todo en destinos europeos como Alemania o Inglaterra, por la flexibilidad de éste a la hora de ofrecer un servicio "puerta a puerta". Además de las preferencias de los operadores, hay otras razones que explican esta situación, por ejemplo, la carencia de una infraestructura que una el transporte marítimo con los modos de superficie y elimine las restricciones derivadas de la ruptura de las cadenas de tránsito (Pérez y Salinas, 2010). En este sentido, las soluciones de transporte intermodal pueden hacer que el tiempo de tránsito sea excesivo o que el producto sea dañado en los transbordos. Sin embargo, estos obstáculos pueden ser gestionados, por lo que el transporte intermodal presenta un potencial alto de crecimiento.

Sin embargo, a nivel europeo, existen conexiones férreas y marítimas que permiten el transporte de todo tipo de mercancías por el continente y las mejoras tecnológicas permiten la existencia de casos de éxito en la logística de mercancías perecederas. Respecto al TMCD, los requisitos mínimos que facilitan el éxito del tránsito para estos productos son (García-Barranco y Pérez-Mesa, 2015):

- Grupaje de cargas para reducir el coste por tránsito, unido a asegurar cargas mínimas para garantizar un tráfico regular.

- Se requiere la organización de los operadores en origen para completar cargas.

- La participación del cliente no es indispensable, ya que existen opciones varias de almacenaje de cargas en los puertos.

La causa principal que dificulta la utilización de este sistema es el retraso y la aleatoriedad en los plazos de entrega, circunstancia que también se repite en el transporte ferroviario.

En resumen, la intermodalidad presenta posibilidades futuras por la saturación del transporte por carretera. Racionaliza la cadena logística de transporte a la par que reduce el consumo de energía, promueve el adecuado uso de las infraestructuras y disminuye el impacto medioambiental aprovechando la capacidad del transporte marítimo y la mayor flexibilidad del de carretera. Además, las políticas públicas y las iniciativas privadas van encaminadas a la promoción de modos alternativos al uso del camión. Ante la falta de desarrollo de las vías ferroviarias que rodean Almería y las ventajas del TMCD, la opción intermodal con prioridad del transporte marítimo es la alternativa más factible desde el sudeste español. 


\section{METODOLOGÍA}

La intermodalidad es una solución eficiente y sostenible frente al transporte por carretera siempre que los costes generales sean iguales o inferiores a este, y se garanticen tiempos y condiciones que aseguren la calidad final de los perecederos. En este trabajo, la opción intermodal priorizando el transporte marítimo es la alternativa estudiada.

De los diferentes buques que realizan las operaciones básicas del TMCD, el tipo Ro-Ro (roll-onroll-off, en el cual el barco transporta la carga sobre ruedas, utilizando una rampa para carga y descarga) son los más adecuados para distancias marítimas cortas, como en el caso de este trabajo. A pesar de un mayor coste respecto a los sistemas de carga vertical (buques Lo-Lo), permiten seguir utilizando el camión refrigerado de origen, tienen servicio regular con mayores frecuencias y alcanzan velocidades marítimas más elevadas (Palao y Salinas, 2007).

La mayoría de los trabajos de viabilidad de rutas intermodales contemplan el estudio de los umbrales mínimos de carga, la predisposición del usuario final, la determinación óptima de los destinos y la comparación en las variables claves de decisión (coste y tiempo) con respecto al transporte base, en este caso camión (Pérez-Mesa y Galdeano-Gómez, 2012; López et al., 2015; Agamez-Arias y MoyanoFuente, 2017). Por esta razón, se realizan cuatro análisis:

i) Estudio de la propensión de implantación: orientado a comprobar si existe un interés real por la problemática en las empresas agroalimentarias almerienses. Se determina a partir de una encuesta a 12 comercializadoras, con un volumen comercializado conjunto de 235.000 toneladas.

ii) Predicción del tránsito futuro de mercancías: definiendo el volumen a transportar por la Fachada Atlántica y la Mediterránea. Se calcula a partir de datos de exportación de ICEX, aceptando que las áreas más pobladas son las que tienen mayor capacidad de compra y asumiendo parte de las exportaciones de Granada (20\%) y Murcia (10\%) por formar parte del hub almeriense.

iii) Determinación de puntos finales de descarga óptima: a partir de la búsqueda de operadores y el análisis de rutas existentes, utilizando como material los informes de las principales Autoridades Portuarias y de las navieras que ofrecen estos servicios.

iv) Análisis de las barreras económicas, fisicas y ambientales: calculando costes, tiempos de tránsito e impacto ambiental en la situación actual (vía terrestre por camión refrigerado) y la potencial (uso intermodal terrestre-marítimo). Se utiliza como herramienta el simulador de cadenas de transporte dentro de la plataforma CoModalWeb 2.0, diseñado por el Ministerio de Fomento en colaboración con la Asociación Española para la Promoción del Transporte Marítimo de Corta Distancia, la Universidad de Cantabria, la Escuela Europea de Short Sea Shipping y las Autoridades Portuarias de los principales puertos españoles.

\section{RESULTADOS Y DISCUSIÓN}

\subsection{Propensión de implantación}

Del análisis de las encuestas se obtiene que el tiempo medio de transporte de la mercancía es de 47 \pm 10 horas; asumiendo y gestionando la propia empresa almeriense una media del $31 \pm 10 \%$ de los tráficos, siendo el resto de los portes gestionados por el cliente.

Aunque el transporte marítimo había sido utilizado alguna vez por el $25 \%$ de los encuestados, su uso de media en campaña se estableció en menos del $2 \%$. En este sentido, las comercializadoras que nunca habían utilizado el transporte marítimo respondieron que la principal razón era que no se garantizaba que el producto llegara a tiempo. Los otros dos motivos más importantes son el desconocimiento del sistema y que consideran que no se garantiza la conservación de la calidad (Figura 3). 


\section{Figura 3}

Motivos por los que no se utiliza transporte marítimo según encuestados

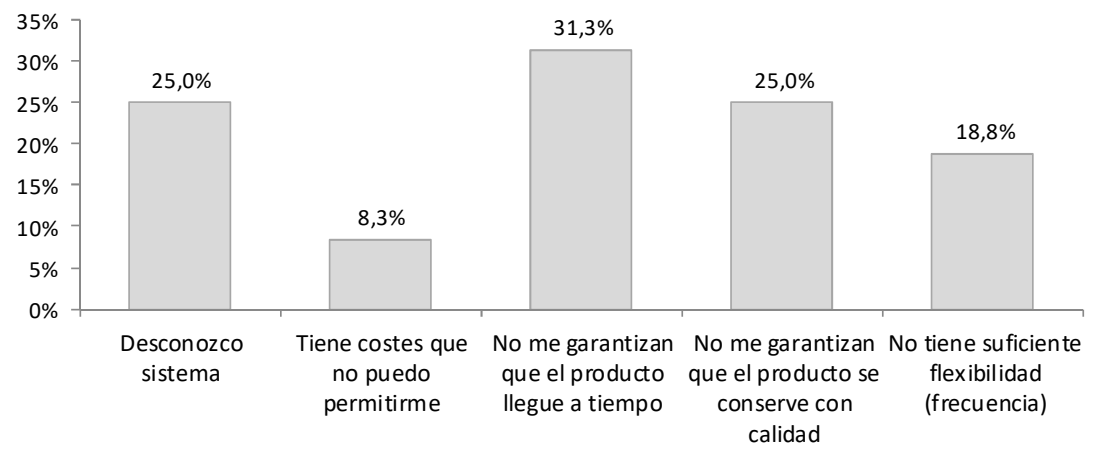

Por otro lado, el factor más importante a la hora de elegir un sistema de transporte es que el envío se haga en el menor tiempo posible, seguido de que los costes sean los más bajos (Tabla 1). Además de los evaluados, los entrevistados describieron otros tres factores: la frecuencia del medio que repercute en el tiempo, la disponibilidad en tiempo real del medio de transporte y que no hay alternativas reales al camión.

Finalmente, el 100\% de los encuestados manifestó su disposición a asociarse temporalmente con otras empresas para hacer envíos en conjunto utilizando nuevas opciones de transporte.

Las encuestas demuestran que aunque el cambio modal puede ser liderado por las comercializadoras, debe hacerse en conjunto con sus principales clientes, las grandes distribuidoras europeas, puesto que son quienes realmente gestionan la mayoría de los portes. Además, acorde a la bibliografía, el transporte marítimo prácticamente no es utilizado, por una cuestión de tiempo.

En cuanto a los criterios para elegir un sistema de transporte, asegurar tiempos y costes son los factores más importantes. Sin embargo, se observa una contradicción en el sector, ya que mientras que las políticas europeas y las tendencias de consumo priorizan cada vez más la sostenibilidad medioambiental, este es el criterio menos valorado por las comercializadoras encuestadas.

En conjunto, las encuestas manifiestan una predisposición hacia nuevas formas de transporte mientras se aseguren tiempos de envío y costes y se acompañe de formación.

Tabla 1

Factores para elegir un sistema de transporte, según encuestados

\begin{tabular}{lcc}
\hline & MEDIA & DESV. TIP \\
\hline El envío se hace en el menor tiempo de entrega posible & 4.6 & 0.5 \\
\hline Los costes son los más bajos & 4.0 & 1.1 \\
\hline $\begin{array}{l}\text { Se mantiene la calidad de los productos enviados de } \\
\text { acuerdo a los estándares de producción y la regulación del } \\
\text { país de destino }\end{array}$ & 2.5 & 1.1 \\
\hline $\begin{array}{l}\text { Se utiliza un medio y servicio solicitado por el cliente } \\
\text { Se utilizan los medios de transporte más respetuosos con el } \\
\text { medioambiente }\end{array}$ & 2.4 & 0.8 \\
\hline
\end{tabular}

Valoración del 1 al 5 donde 5 es el más importante y 1 el que menos. 


\subsection{Predicción del tránsito de mercancías}

Existiendo una predisposición por parte de los miembros del sector, para conocer la viabilidad de la propuesta es necesario calcular la actual demanda hortofrutícola procedente de Almería en las vertientes Atlántica y Mediterránea. Por un lado, los países prioritarios son aquellos que desde hace años son los mayores importadores de productos almerienses, es decir, Alemania, Francia, Reino Unido, Países Bajos e Italia. Por otra parte, hay una zona secundaria de países complementarios que aunque importan una menor cuota pueden verse beneficiados de las líneas intermodales por su proximidad y buenas conexiones con la zona prioritaria: Bélgica, Suiza, Austria e Irlanda (Figura 4). Dada la necesidad de añadir un segundo buque una vez llegado a Reino Unido y debido a los bajos volúmenes que se demandan, se descartó considerar Irlanda dentro de la zona de influencia británica a efectos de cálculo.

Figura 4

Localización de la demanda hortofrutícola almeriense en Europa Occidental

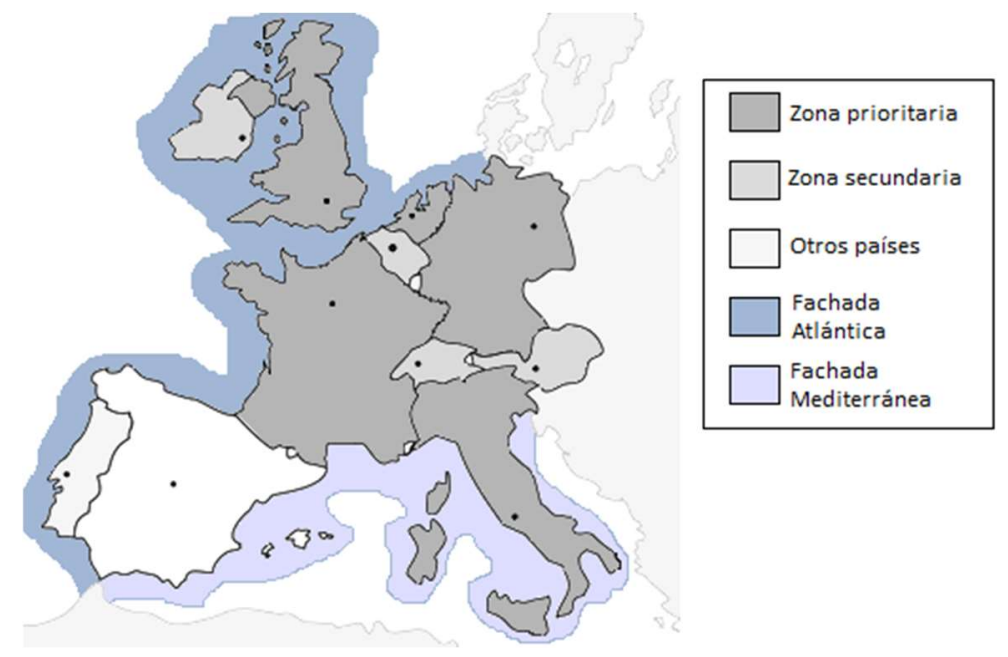

Fuente: Elaboración propia.

En función de la densidad de población de estos países, se estima la demanda final (o el consumo o aprovisionamiento necesario), asumiendo que las zonas más pobladas son las que poseen mayor capacidad de compra. Se distingue entre la fachada mediterránea y atlántica y se considera que Reino Unido tiene una demanda suficiente para utilizar una línea directa con la que evitar la utilización de un segundo buque desde el primer puerto, ahorrando en tiempo. Además, se contempla la posibilidad de que se comercialice el 20\% de las exportaciones de Granada y el 10\% de Murcia. Teniendo en cuenta las zonas a abastecer, los volúmenes y la producción que se asume, se obtiene la estimación del posible tránsito (Tabla 2).

Tabla 2

Estimación de tránsito según destino. Año 2017. Toneladas

\begin{tabular}{lrrr}
\hline & \multicolumn{2}{c}{ Zona atlántica } & \multicolumn{2}{c}{ Zona mediterránea } \\
\hline $\begin{array}{l}\text { Tránsito } \\
\text { estimado } \\
\text { (Toneladas) }\end{array}$ & $\begin{array}{l}100 \% \\
\text { Reino }\end{array}$ & $\begin{array}{c}60 \% \text { Francia }+75 \% \text { Alemania }+ \\
100 \% \text { (Bélgica + Países Bajos) }\end{array}$ & $\begin{array}{c}40 \% \text { Francia }+25 \% \text { Alemania }+ \\
100 \% \text { (Italia + Suiza + Austria) }\end{array}$ \\
\hline Almería (100\%) & 280 & 1117474 & 539220 \\
\hline Granada (20\%) & 3390 & 21351 & 6128 \\
\hline Murcia (10\%) & 39573 & 75313 & 34473 \\
\hline Total & $\mathbf{3 2 3}$ & $\mathbf{1 2 1 4} \mathbf{1 3 8}$ & $\mathbf{5 7 9 8 2 0}$ \\
\hline
\end{tabular}

Las tres grandes áreas que se han seleccionado tienen capacidad para distribuir el $82,3 \%$ de las actuales exportaciones de Almería, el 8,4\% de las de Murcia y el 15,6\% de Granada. 


\subsection{Determinación de puntos finales de descarga óptima.}

Se entiende que el punto final de descarga óptima en cada una de las tres zonas seleccionadas (según Figura 4) debe estar en una posición estratégica con conexiones cortas hacia las distintas áreas de demanda. Con este criterio, se limita la búsqueda de operadores al sur y centro de Inglaterra para Reino Unido, al norte de Francia, Bélgica o Países Bajos en la fachada atlántica; y al noreste de Italia en la vertiente mediterránea.

Por otro lado, de los diferentes puertos que operan con buques Ro-Ro, los más importantes son Santander, Bilbao y Vigo en la fachada atlántica, y Barcelona y Valencia en la mediterránea.

Analizando las posibles rutas desde estos puertos hacia las zonas de descarga óptima establecidas, buscando las mayores frecuencias, los mejores precios entre navieras y las menores distancias terrestres, se seleccionan tres puertos de descarga:

- Hacia Reino Unido, Portsmouth (Inglaterra) vía Bilbao.

- En la fachada atlántica, Le Havre (Francia) vía Santander.

- En la fachada mediterránea, Savona (Italia) vía Valencia.

\subsection{Análisis de barreras económicas, físicas y ambientales.}

Se distinguen cinco rutas por el océano Atlántico (de las cuales una es directa hacia Reino Unido) y otras cinco por el mar Mediterráneo (Tabla 3, Figura 5).

Tabla 3

Rutas seleccionadas para su análisis desde la vertiente atlántica y mediterránea

\begin{tabular}{|c|c|c|c|c|c|c|c|}
\hline & & \multicolumn{4}{|c|}{ Intermodal } & \multicolumn{2}{|c|}{ Carretera } \\
\hline \multirow{5}{*}{ 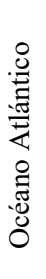 } & REINO UNIDO & Almería & Bilbao & Portsmouth & Londres & Almería & Londres \\
\hline & ALEMANIA & Almería & Santander & Le Havre & Berlín & Almería & Berlín \\
\hline & FRANCIA & Almería & Santander & Le Havre & París & Almería & París \\
\hline & $\begin{array}{l}\text { PAÍSES } \\
\text { BAJOS }\end{array}$ & Almería & Santander & Le Havre & Ámsterdam & Almería & Ámsterdam \\
\hline & BÉLGICA & Almería & Santander & Le Havre & Bruselas & Almería & Bruselas \\
\hline & ALEMANIA & Almería & Valencia & Savona & Múnich & Almería & Múnich \\
\hline 胥 & FRANCIA & Almería & Valencia & Savona & Marsella & Almería & Marsella \\
\hline 兽 & ITALIA & Almería & Valencia & Savona & Roma & Almería & Roma \\
\hline$\sum_{i=1}$ & AUSTRIA & Almería & Valencia & Savona & Viena & Almería & Viena \\
\hline$\Sigma$ & SUIZA & Almería & Valencia & Savona & Berna & Almería & Berna \\
\hline
\end{tabular}

Figura 5

Ejemplo de rutas seleccionadas para su comparativa

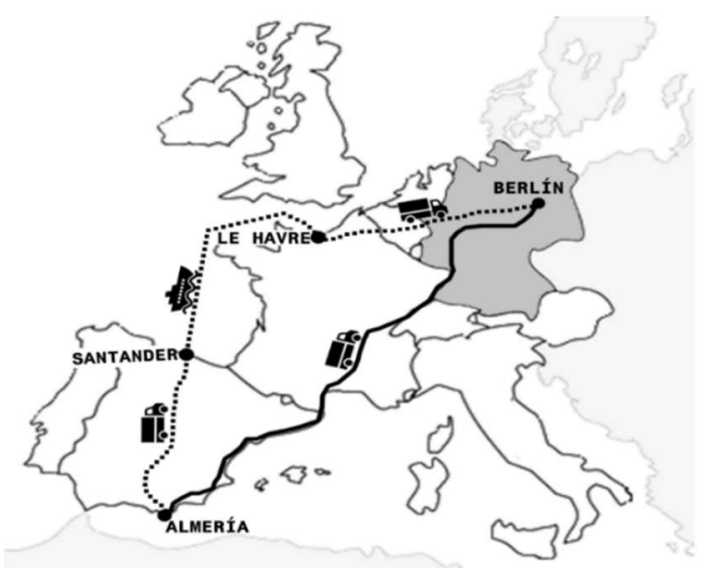


Para cada ruta, en el simulador se consideran 18 toneladas netas de carga refrigerada en semirremolques vía terrestre y vía marítima con buques Ro-Ro. Además, se tiene en cuenta que en el vehículo sólo va un conductor y se calcula el tiempo de cada trayecto respetando tanto el descanso diario como las pausas obligatorias en la conducción diaria.

Los parámetros que se deben definir para el simulador son: i) los costes en $€ / \mathrm{km}$, calculados a partir del coste nominal del año 2018 para un vehículo frigorífico de doble eje, según el Ministerio de Fomento, y ii) la velocidad media para los tramos terrestres en $\mathrm{km} / \mathrm{h}$, obtenidos a partir de la Guía de seguridad vial de la Unión Europea ${ }^{\bullet}$, priorizando el transporte vía autovía frente a autopista, para no considerar peajes, y aplicando una reducción del $5 \%$ a la velocidad máxima de la vía para este tipo de vehículos, asumiendo pérdidas a lo largo del trayecto (por la utilización de vías secundarias, incorporaciones, pendientes, entre otras).

Los resultados obtenidos fueron analizados distinguiendo entre la fachada atlántica y la mediterránea (Tabla 3).

Tabla 3

Comparativa de la ruta potencial marítimo-terrestre frente a la actual para cada país de destino

\begin{tabular}{cccccc}
\hline \multicolumn{7}{c}{ Océano Atlántico } & Coste & \multicolumn{2}{c}{ Tiempo } & Distancia & Emisión CO2 \\
\hline BÉLGICA & $+10.2 \%$ & $+26.4 \%$ & 12.9 horas & $+12.7 \%$ & $-29.3 \%$ \\
\hline PAÍSES BAJOS & $+9.3 \%$ & $+1.9 \%$ & 1.2 horas & $+11.3 \%$ & $-27.1 \%$ \\
\hline FRANCIA & $+18.5 \%$ & $+31.6 \%$ & 14.1 horas & $+20.6 \%$ & $-28.6 \%$ \\
\hline ALEMANIA & $+18.2 \%$ & $+21.6 \%$ & 14.7 horas & $+18.7 \%$ & $-15.1 \%$ \\
\hline REINO UNIDO & $-26.0 \%$ & $+6.9 \%$ & 3.5 horas & $-8.4 \%$ & $-48.4 \%$ \\
\hline País & & Mar Mediterráneo & & \\
\hline AUSTRIA & Coste & \multicolumn{2}{c}{ Tiempo } & Distancia & Emisión CO2 \\
\hline SUIZA & $-17.0 \%$ & $16.5 \%$ & 10.8 horas & $-9.9 \%$ & $-39.8 \%$ \\
\hline FRANCIA & $-9.1 \%$ & $30.1 \%$ & 13.4 horas & $+3.3 \%$ & $-41.8 \%$ \\
\hline ALEMANIA & $+14.0 \%$ & $103.3 \%$ & 28.3 horas & $+29.6 \%$ & $-30.8 \%$ \\
\hline ITALIA & $-18.2 \%$ & $19.1 \%$ & 9.7 horas & $-8.5 \%$ & $-44.4 \%$ \\
\hline
\end{tabular}

Respecto a la vertiente atlántica, las rutas intermodales estudiadas hacia Europa Occidental tienen un coste un 9-18\% más alto que utilizando sólo el transporte por carretera y el tiempo de tránsito se incrementa en un promedio de 10 horas. Sin embargo, la ruta intermodal directa a Reino Unido es un $26 \%$ más barata y el tiempo de tránsito se incrementa sólo en 3.5 horas respecto a camión pero contaminando casi un 50\% menos. En el resto de líneas por el Atlántico, la opción intermodal reduce la emisión de $\mathrm{CO} 2$ en torno a un 25\%. En conjunto, la ruta marítimo-terrestre hacia Reino Unido es la única con opciones reales de implementación, puesto que es más económica y el incremento en las horas de tránsito no es significativo (Figura 6).

\footnotetext{
• En red: http://ec.europa.eu/transport/road_safety/going_abroad/index_es.htm
} 


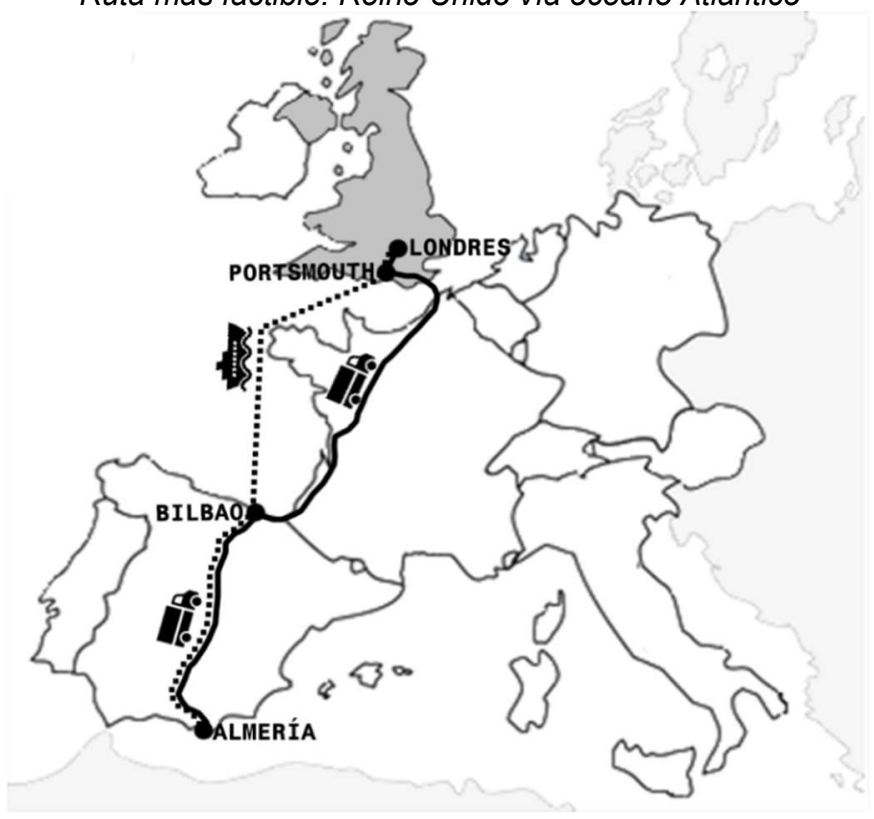

\begin{tabular}{|c|c|c|c|c|c|}
\hline & & Coste $(€)$ & $\begin{array}{l}\text { Tiempo } \\
\text { (horas) }\end{array}$ & $\begin{array}{c}\text { Distancia } \\
(\mathbf{k m})\end{array}$ & $\begin{array}{c}\text { Emisión CO2 } \\
(\mathrm{kg})\end{array}$ \\
\hline \multicolumn{2}{|l|}{ Carretera } & 3698 & 50.5 & 2282 & 5053 \\
\hline \multirow{4}{*}{$\begin{array}{l}\text { Intermodal } \\
\ldots\end{array}$} & I & 1479 & 22.7 & 942 & 2085 \\
\hline & II & 1060 & 30 & 1028 & 257 \\
\hline & III & 197 & 1.3 & 120 & 265 \\
\hline & TOTAL & 2736 & 54 & 2090 & 2607 \\
\hline \multicolumn{2}{|c|}{ Variación } & $-26.01 \%$ & $6.93 \%$ & $-8.41 \%$ & $-48.41 \%$ \\
\hline
\end{tabular}

En relación con la vertiente mediterránea, la ruta intermodal estudiada hacia el sur de Francia no es rentable ni en costes ni en tiempos. Sin embargo, el resto de rutas son entre un 10-22\% más baratas aunque con un incremento de tiempo promedio de 11 horas. En este caso, las emisiones de CO2 se reducen entre un 40-50\% con la opción intermodal (sin considerar Francia). De las rutas estudiadas por esta fachada, la intermodal hacia Italia es la mejor por diferencia de costes y reducción de $\mathrm{CO} 2$, pero aumenta el tránsito en 10 horas.

En todas las situaciones, hay que resaltar que la diferencia de costes se haría más pequeña introduciendo peajes y costes externos (derivados de accidentes, congestión...), que incrementarían el tránsito en camión en torno a un 5-10\%, dependiendo de la ruta. También, la adición de un segundo conductor reduciría el tiempo de los tramos terrestres mientras que la gestión del retorno del camión lleno de vuelta a Almería reduciría los costes de transporte y supone una opción más sostenible que el retorno vacío.

Dentro de las Directrices Nacionales para la elaboración de los pliegos de condiciones referentes a las acciones medioambientales de las Organizaciones de Productores de Frutas y Hortalizas, la forma jurídica en la que se agrupan la mayoría de comercializadoras almerienses según la normativa comunitaria, se incluye un apartado ${ }^{\bullet}$ por el cual se pueden recibir ayudas por el total del diferencial de costes entre el transporte por ferrocarril o marítimo generado en el año en cuestión y el coste de transporte en carretera generado en la media de los dos años anteriores.

\footnotetext{
- Acción 7.26. Año 2017. En red: https://www.mapama.gob.es/es/agricultura/temas/regulacion-de-losmercados/directricesmedioambientales2017_tcm30-380318.pdf
} 


\section{CONCLUSIONES}

La intermodalidad se configura como una solución a la saturación del transporte por carretera. Racionaliza la cadena logística de transporte, reduce el consumo de energía, promueve el adecuado uso de las infraestructuras y disminuye el impacto medioambiental. Este sistema aprovecha la amplia capacidad de carga del transporte marítimo y la flexibilidad de la carretera; además está perfectamente alineado con las políticas públicas y las iniciativas privadas que promueven modos alternativos al uso del camión. Ante la falta de desarrollo de las conexiones ferroviarias, la opción intermodal, con prioridad del transporte marítimo, es la alternativa más factible del sudeste español para ampliar sus alternativas logísticas.

De forma concreta, se observa que existe una predisposición por parte de las comercializadoras hortícolas al cambio modal siempre que se aseguren los tiempos y costes del transporte actual por carretera. Sin embargo, aunque las líneas intermodales son significativamente más sostenibles (entre un 15-50\%), en líneas generales no igualan ni en tiempos ni en costes al tráfico terrestre.

En conjunto, el cambio modal pasa no sólo por subvencionar estas diferencias de coste, sino por apoyar e incentivar a las comercializadoras que apuesten hoy en día por una logística más sostenible. A largo plazo, mayores volúmenes supondrán mejores precios de las navieras, mayores frecuencias e incluso la disponibilidad de buques con mayores velocidades con los que reducir los tránsitos.

La ruta intermodal desde Almería a Reino Unido por los puertos de Bilbao y Portsmouth es la única opción factible hoy por hoy: se consigue un ahorro de costes del $26 \%$ con diferencias de tiempo asumibles. Desde el punto de vista medioambiental, el cambio modal del 100\% del tráfico estimado a Reino Unido emitiría 43 millones de toneladas menos de $\mathrm{CO} 2$ a la atmósfera que la opción actual. Por otro lado, estos resultados son relevantes en cuanto que es previsible que el tráfico terrestre a Reino Unido sufra una ralentización muy elevada como consecuencia del establecimiento de controles fronterizos derivados del BREXIT. La utilización del TMCD en un marco intermodal podría aliviar esta situación.

\section{REFERENCIAS BIBLIOGRÁFICAS}

AGAMEZ-ARIAS, A. y MOYANO-FUENTE (2017): "Intermodal transport in freight distribution: a literature review", en Transport Reviews, Vol. 37(6), pp. 782-807.

BOER, E. D.; ESSEN, H. V.; BROUWER, F.; PASTORI, E.; y MOIZO, A. (2011): Potential of modal shift to rail transport - Study of the projected effects on GHG emissions and transport volumes. Delft: Community of European Railway and Infrastructure Companies (CER).

CAJAMAR (2017): Análisis de la campaña hortofrutícola de Almería. Campaña 2016-2017. Almería: Fundación Cajamar.

COMISIÓN EUROPEA (2001): "Libro blanco del transporte. La política europea de transportes de cara al 2010: la hora de la verdad". Disponible en: https://ec.europa.eu/transport/sites/transport/files/themes/strategies/doc /2001_white_paper/lb_com_2001_0370_es.pdf [10/10/2018].

COMISIÓN EUROPEA (2011): "Libro blanco del transporte. Hoja de ruta hacia un espacio único europeo de transporte". Disponible en: https://ec.europa.eu/transport/sites/transport/files/themes/strategies/doc/2011 white_paper/white-paper-illustrated-brochure_es.pdf [20/10/2018].

COM̄MISIÓN EUROPEA (2018): "Transport in the European Union: Current Trends and Issues". Disponible en: https://ec.europa.eu/transport/sites/transport/files/2018-transport-in-the-eu-current-trends-and-issues.pdf [12/10/2018].

EUROSTAT (2017): "Estadísticas de transporte". Disponible en: http://ec.europa.eu/eurostat. [12/09/2018].

GARCÍA-BARRANCO, M. C. y PÉREZ-MESA, J. C. (2015): Influencia de la distribución europea en la gestión logística del exportador. El caso de España, Alemania, Francia y Países del Este. Almería: Editorial Universidad de Almería y Cátedra de Logística de Andalucía.

GARCÍA-BARRANCO, M. C.; PÉREZ-MESA, J. C. y GALDEANDO-GÓMEZ, E. (2016): "El transporte como factor limitante de crecimiento de las exportaciones de perecederos", en Tribuna de Economía, ICE, núm. 888, pp. 155-163.

HANSSEN, T.; MATHISEN, T. y JORGENSEN, F. (2012): "Generalized transport costs in intermodal freight transport", en Procedia - Social and Behavioral, núm. 54: pp. 189-200.

ICEX (2017): "Datos de exportación". Disponible en: https://www.icex.es/icex/es/index.html [01/10/2018].

LÓPEZ, A. M.; KRONBAK, J. y LIPING, J. (2015): "Cost and time models for the evaluation of intermodal chains by using short sea shipping in the North Sea Region: the Rosyth-Zeebrugge route", en International Journal of Shipping and Transport Logistics, Vol. 7(4), pp. 494-520

MEDDA, F. y TRUJILLO, L. (2010): "Short-sea shipping: An analysis of its determinants", en Maritime Policy \& Management, vol. 37(3), pp. 285-303. 
MINISTERIO DE FOMENTO (2005): "El plan estratégico de infraestructuras y transporte 2005-2020". Disponible en: https://www.fomento.es/plan-estrategico-de-infraestructuras-y-transporte-peit [05/10/2018].

MINISTERIO DE FOMENTO (2017): "Observatorio de mercado del transporte de mercancías por carretera”, marzo 2018. Disponible en: https://www.fomento.gob.es/recursos_mfom/comodin/recursos/observatoriomercadon 27marzo2018.pdf [20/09/2018].

PAIXAO, A. C. y MARLOW, P. B. (2009): "Logistics strategies for short sea shipping operating as part of multimodal transport chains", en Journal of Maritime Policy \& Management, Vol. 36(1), pp. 1-19.

PALAO, F. y SALINAS, J. A.; (2007): Potencialidad del transporte hortofrutícola por el Puerto de Almería. Almería: Editorial Universidad de Almería.

PÉREZ, J.C. y SALINAS, J. A. (2010): "Distribución óptima del transporte intermodal: aplicación a la exportación de perecederos", en Economía Agraria y Recursos Naturales, núm. 10, pp. 93-119.

PÉREZ-MESA, J.C. y GALDEANO, E. (2012): "Logistics network and externalities for short sea transport: An analysis of horticultural exports from southeast Spain", en Transport Policy, vol. 24, pp. 188-198.

PÉREZ-MESA, J.C. y GALDEANO, E. (2015). "Collaborative firms managing perishable products in a complex supply network: an empirical analysis of performance”, en Supply Chain Management: An International Journal, vol. 20(2), pp. 128-138

PÉREZ-MESA, J.C.; CÉSPEDES, J. y SALINAS, J. (2010): "Feasibility study for a Motorways of the Sea from Almeria (Spain) to Port Vendres-Perpignan (France). Application to the transportation of fruit and vegetables", en Transport Review, vol. 30(4), pp. 451-471.

SERRANO-ARCOS, M. (2018): "Product-country image and crises in the Spanish horticultural sector", en International Food Marketing Research Symposium. Bournemouth (UK): 13-14 June.

SUÁREZ-ALEMAN, A. (2016): "Short sea shipping in today's Europe: A critical review of maritime transport policy", en Maritime Economics \& Logistics, Vol.18(3), pp. 331-351.

SUÁREZ-ALEMÁN, A.; TRUJILLO, L. y MEDDA, F. (2014): "Short sea shipping as intermodal competitor: A theoretical analysis of European transport policies", en Maritime Policy \& Management, vol. 42(4), pp. 1-18. 